\title{
Not all Speed-Accuracy Trade-Off Manipulations Have the Same Psychological Effect
}

\author{
Dimitris Katsimpokis $^{1,2} \cdot$ Guy E. Hawkins ${ }^{3}$ - Leendert van Maanen ${ }^{1,4}(\mathbb{C}$
}

Published online: 28 January 2020

(C) The Author(s) 2020

\begin{abstract}
In many domains of psychological research, decisions are subject to a speed-accuracy trade-off: faster responses are more often incorrect. This trade-off makes it difficult to focus on one outcome measure in isolation - response time or accuracy. Here, we show that the distribution of choices and response times depends on specific task instructions. In three experiments, we show that the speed-accuracy trade-off function differs between two commonly used methods of manipulating the speed-accuracy tradeoff: Instructional cues that emphasize decision speed or accuracy and the presence or absence of experimenter-imposed response deadlines. The differences observed in behavior were driven by different latent component processes of the popular diffusion decision model of choice response time: instructional cues affected the response threshold, and deadlines affected the rate of decrease of that threshold. These analyses support the notion of an "urgency" signal that influences decision-making under some time-critical conditions, but not others.
\end{abstract}

Keywords Diffusion decision model $\cdot$ Decision-making $\cdot$ Time pressure $\cdot$ Decreasing thresholds

\section{Introduction}

Decision-making has been a focus of psychological research for decades. In many decision-making contexts, time pressure is of critical importance. A well-studied decision outcome of time pressure is the speed-accuracy trade-off (SAT): a decision-maker can improve the accuracy of their decisions at the expense of taking longer to respond, or they can make very quick decisions that are more likely to be erroneous (Schouten and Bekker 1967; Wickelgren 1977). This pattern

Code for the models and data are provided at OSF: https://osf.io/r7dzv/

Electronic supplementary material The online version of this article (https://doi.org/10.1007/s42113-020-00074-y) contains supplementary material, which is available to authorized users.

Leendert van Maanen

1.vanmaanen@uva.nl

1 Department of Psychology, University of Amsterdam, P.O. Box 15906, 1001, NK Amsterdam, the Netherlands

2 Department of Psychology, University of Basel, Basel, Switzerland

3 School of Psychology, University of Newcastle, Callaghan, NSW, Australia

4 Amsterdam Brain and Cognition Center, University of Amsterdam, Amsterdam, the Netherlands of results produces the trade-off between speed and accuracy, and it is the task of the decision-maker to balance the relationship between accuracy and speed to achieve their desired level of performance (cf. Bogacz et al. 2006).

A range of experimental manipulations have been used to elicit the SAT in previous research (see Heitz 2014 for an overview). Cue-based SAT is arguably the most frequently used speed-accuracy manipulation. The cues are typically verbal prompts instructing participants to focus on responding correctly or responding speedily (Hale 1969; Howell and Kreidler 1963). Another common SAT manipulation uses response deadlines (Ratcliff and Rouder 2000; Van Zandt et al. 2000). Response deadlines usually involve a visual or auditory message following each trial if the participant did not respond sufficiently fast. In most cases, the deadline is prespecified by the experimenter. Recently, some authors have combined the two SAT manipulations into one design (e.g., Forstmann et al. 2008; Mulder et al. 2013; Wagenmakers et al. 2008). Although both manipulations produce a manifest SAT in behavioral data, ${ }^{1}$ we hypothesize that the cognitive processes invoked by the two manipulations differ. In the current

\footnotetext{
${ }^{1}$ SAT refers here to the empirical phenomenon of lower accuracy when speed is emphasized (and vice versa). This is not to be confused with the use of SAT to describe the latent psychological mechanisms that underlie or cause the observed pattern in data.
} 
paper, we test this hypothesis in three experiments and show that choices and response times differ between cue-induced SAT conditions and deadline-induced SAT conditions. We also show that these differences can be attributed to different latent component processes of the popular diffusion decision model (DDM) of choice response time (Forstmann et al. 2016; Ratcliff 1978; Ratcliff and McKoon 2008; Ratcliff and Rouder 1998).

A standard practice in perceptual decision-making research has been the application of sequential sampling models, also known as evidence accumulation models, to draw inferences about the cognitive processing mechanisms underlying decision-making (Forstmann et al. 2016; Gold and Shadlen 2007; Mulder et al. 2014; Ratcliff and McKoon 2008; Ratcliff et al. 2016). ${ }^{2}$ These models hypothesize that decisions involve accumulation of sensory information in favor or against certain choices at hand. Accumulation stops when enough information has been collected to support some specific choices. The stopping point represents the uncertainty that the decision-maker is willing to accept on the correctness for this particular choice: more lenient stopping points correspond to greater uncertainty.

Within the class of sequential sampling models, the DDM (Ratcliff 1978; Ratcliff and McKoon 2008) is an important tool for isolating and understanding the cognitive processes that underlie decision behavior, because of its demonstrated ability to provide a quantitatively precise account of the response time (RT) distributions of correct/error responses in two-alternative forced choice tasks (Ratcliff and McKoon 2008; Ratcliff and Rouder 1998; Ratcliff and Smith 2004; Ratcliff et al. 2016). To account for time pressure in decision-making, the DDM assumes a set of two thresholds, one for each of the possible response options (Fig. 1, left panel). The decision-maker's willingness to commit to decisions with greater uncertainty is translated into thresholds that are close to the starting point of the accumulation process (green lines). This response regime means that the accumulation process often reaches one of the two thresholds before much accumulation has even taken place; this leads to fast responses that are often erroneous. Conversely, thresholds that sit further from the starting point (blue lines) represent a more conservative decision strategy whereby the decision-maker accumulates a greater amount of evidence before committing to a decision; this leads to slower but more accurate responses. Such changes between low and high thresholds have provided a good explanation of SAT behavior across a range of contexts (Bogacz et al. 2010; Forstmann et al. 2008; Ratcliff and McKoon 2008; Ratcliff and Rouder 1998; Voss et al. 2004; Wagenmakers et al. 2008), although the link between the

\footnotetext{
2 Similar models have also been used to explain preferential decision-making (e.g., Bhatia 2013; Busemeyer et al. 2019; Roe et al. 2001; Trueblood et al. 2014; Turner et al. 2018).
}

computational explanation of SAT and its low-level neural implementation is still a matter of active research (e.g., Heitz and Schall 2012; Reppert et al. 2018).

In contrast to the fixed-threshold adjustment explanation of the cue-used SAT, Frazier and Yu (2008), Cisek et al. (2009), and Thura et al. (2012) gave different but converging explanations about the cognitive processes involved when decisionmakers are faced with deadlines (see also Malhotra et al. 2017). According to Frazier and Yu (2008), a stopping rule in the form of thresholds that dynamically move toward one another (decline) throughout the course of a decision provides a mechanism that ensures the decision-maker responds before a deadline, whether that deadline is internally or externally imposed, in the most efficient way (Fig. 1, right panel). Frazier and $\mathrm{Yu}$ (2008) argue that in the presence of response deadlines, thresholds that monotonically and nonlinearly collapse over time is the optimal decision-making strategy (i.e., the decision policy that maximizes reward over a series of trials). In a similar vein, Cisek et al. and Thura et al. proposed a decision-making model that incorporated an urgency signal that increases with elapsed decision time; this can be viewed as an internally imposed deadline, as the urgency signal monotonically increases the probability of committing to a choice as decision time increases. ${ }^{3}$

Given that the collapsing thresholds and urgency signal models on the one hand, and DDMs with fixed thresholds on the other, make different quantitative behavioral predictions about choice and RT distributions (for a review see Hawkins et al. 2015b), the empirical validity of these contrasting theoretical accounts have been systematically studied by a variety of researchers (Evans et al. 2017; Ratcliff and Smith 2004; Van Zandt et al. 2000; Winkel et al. 2014). Although previous research has shed light on the way deadlines and cues affect core empirical measures of decision-making, such as the shape of RT distributions, and how these may be attributed to different psychological processes (Evans et al. in press), to our knowledge, the empirical effects of cue-based and deadlinebased manipulations of the SAT have not been directly compared within the same experimental design. Such a direct comparison would make it possible to control for both SAT manipulations (cue, deadline) and to generalize their effects across different tasks. Furthermore, directly comparing these manipulations within the same design would allow us to propose a model-based account for the differential effects of the two SAT manipulations on the choice-RT distributions. This will contribute to the debate surrounding the nature of response thresholds (fixed vs. decreasing) in evidence accumulation models of decision-making under time pressure.

\footnotetext{
${ }^{3}$ Beyond the SAT, collapsing thresholds might arise for different reasons. For example, there may be limited capacity for evidence to become saturated over time, as in recognition decisions (e.g., Cox and Shiffrin 2017).
} 


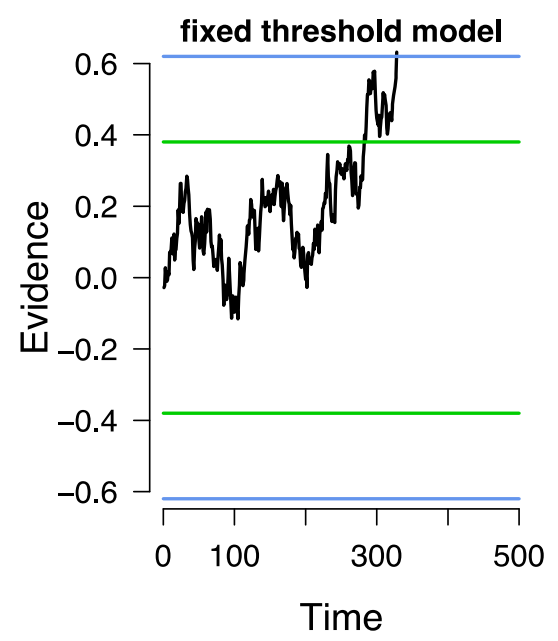

Fig. 1 Different assumptions in diffusion models. Left panel: the standard diffusion decision model (DDM) assumes thresholds that are fixed throughout the course of a decision and only vary with respect to the distance between lower and upper threshold. Green thresholds indicate a speedy response regime; blue thresholds indicate a more accurate

To that goal, we performed three behavioral experiments that each factorially crossed cue-based and deadline-based manipulations of the SAT in a within-subject design to determine whether they led to differential effects on observed choices and response times. Our central aim was to examine the cognitive processes that distinguish performance in cuebased and deadline-based SAT manipulations (Section 5). In particular, we accounted for decision processes in terms of evidence accumulation, where the threshold of the accumulation process was expected to discriminate between the two forms of SAT manipulation, by attributing the differential effects of cues and deadlines to distinct components of strategic adjustments to response caution. Our analyses included a systematic model comparison of DDMs with fixed and decreasing threshold parametrizations encoding different ways of adjusting response caution to establish which adjustment mechanisms better explain decision processes under different forms of time pressure.

\section{Methods}

\section{Participants}

Twenty-four participants affiliated with the University of Amsterdam participated in Experiments 1 and 2 (mean age $=$ 23.45 , SD age $=7.86,58 \%$ female) in the same experimental session. A different sample of twenty-four participants affiliated with the University of Amsterdam participated in Experiment 3 (mean age $=22.75$, SD age $=3.40,62 \%$ female). All participants provided informed consent prior to participation and chose between a monetary or research credit reward at will.

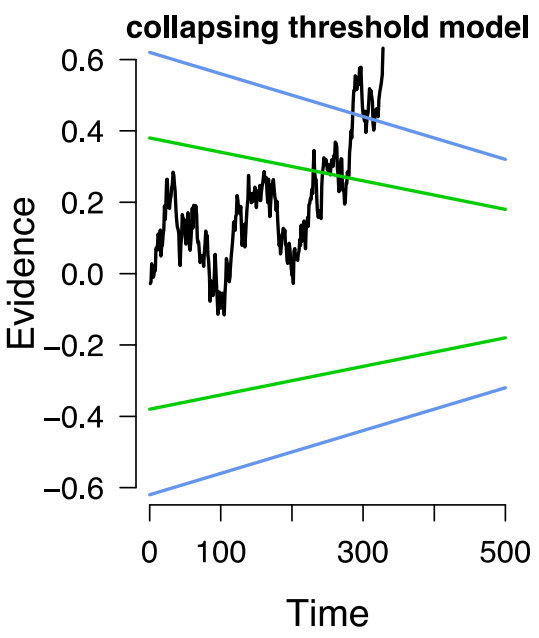

response regime. Right panel: DDM with collapsing thresholds where the slope indicates the amount of evidence required to make a decision as a function of elapsed decision time, and distance between the thresholds at time 0 indicates the initial response caution

\section{Design and Procedure}

\section{Experiment 1}

Participants made motion direction judgments about randomdot kinematograms (Ball and Sekuler 1982). In a random dot motion task, participants are presented with a cloud of dots, a subset of which move coherently in a particular direction, while the remaining dots move in random directions. The participant's task is to determine the direction of the coherently moving dots. The experiment was implemented with the default version of the Random dot motion task in PsychoPy (Peirce 2007) for which the default random dot kinematic component was used (Scase et al. 1996). The settings were dot life-time of 5 frames, dot size of 4 pixels, cloud-size of 400 dots, and speed of dots of $0.5 \%$ of monitor dimensions per frame and the so-called position movement algorithm from PsychoPy. The experiment was conducted on a desktop computer with stimuli presented on a 21 -in. monitor set at $60 \mathrm{~Hz}$ frame rate.

The task consisted of two phases: training and test. The aim of the training phase was to familiarize participants with the task and to establish settings for the test phase. Task instructions were provided on screen with participants briefly introduced to the structure of the task and given instructions to focus on the accuracy of their responses. Keyboard keys were used to make a response ("f" to indicate the dots move left, to the " $\mathrm{j}$ " to indicate dots move to the right). A trial finished with the participant's response and feedback was given based on response accuracy.

In the training phase, participants made decisions across 6 difficulty levels where difficulty was manipulated by changing the percentage of coherently moving dots: $3 \%, 7 \%, 11 \%$, 
$15 \%, 19 \%$, and $23 \%$. There were 40 trials for each difficulty level for a total of 240 trials. Following completion of the training phase, a single difficulty level was selected separately for each participant for use in their test phase. This value was selected as the lowest motion coherence at which a participant scored above $80 \%$ in the training session.

The test phase had a $2 \times 2$ within-subjects design. The first factor was whether participants were cued to respond fast or accurately. The second factor was whether there was a response deadline (i.e., an upper limit on the available decision time) or not. This produced four conditions in the experiment: speed cue with deadline, speed cue without deadline, accuracy cue with deadline, and accuracy cue without deadline. The four conditions were presented in separate blocks with block order counterbalanced across participants. Table 1 shows the task instructions given at the beginning of each block for the four conditions. There were 200 trials in each of the 4 conditions/blocks for a total of 800 trials in the test phase.

The cue-based SAT manipulation was operationalized by giving instructions prior to each block (Table 1). The deadline manipulation was operationalized as a trial cut-off at $1.35 \mathrm{~s}$, which was selected based on pilot data. If a response had not been registered by the time of the deadline, then the stimulus was removed from the display, and responses were no longer accepted. No trial cutoff was present in blocks without deadlines.

The pre-stimulus period (fixation cross, $250 \mathrm{~ms}$ ) directed focus to the center of the screen, after which the stimulus was presented. The stimulus duration then depended on the condition. In the no-deadline condition, the stimulus remained on the screen until the participant gave a response. In the deadline condition, the stimulus was presented at most for $1.35 \mathrm{~s}$, which was the deadline cut-off. If participants missed the deadline, the trial was terminated immediately. Otherwise, by giving a response, the participant ended the trial.

To increase the likelihood that participants followed the block-based instructions, they received feedback following each trial tailored to the pair of conditions in the current block. The text that was given as feedback is shown in Table 1. In the deadline condition, feedback was based on whether the response was prior to the deadline or not. In the condition without a deadline, there was no feedback. In the accuracy cue condition, the feedback focused on whether the response was correct or not. In the speed cue condition, to ensure that participants did not associate feedback related to the cue with a specific cut-off value (i.e., as a deadline), we used a probabilistic feedback method. The feedback stated whether the response was fast or not fast. The probability of "not fast" feedback increased with the trial RT, according to a cumulative Gamma distribution fit to each participant's RT data from the training phase. Thus, the slower a response, the more likely it was the participant would receive "not fast" feedback, but in an individualized fashion. Because feedback was probabilistic, there was no duration after which participants were guaranteed to receive "not fast" feedback, which would essentially implement a response deadline. For all conditions, the feedback duration varied with respect to being positive or negative; positive feedback was shown for $250 \mathrm{~ms}$, and negative feedback was shown for $1 \mathrm{~s}$.

\section{Experiment 2}

To generalize the results of Experiment 1 to a different decision environment, we used a rapid expanded judgment task where participants were presented with two flashing circles and they were asked which of the two circles has the higher flashing rate (cf. Brown et al. 2009; Hawkins et al. 2012; Smith and Vickers 1989); we refer to this as the flash task. The flash task that externalizes to the stimulus display a discrete and explicit evidence process, which we assume gives rise to a corresponding internal evidence accumulation process. This contrasts to the internalized evidence accumulation process that is assumed in most perceptual decision-making tasks, such as random dot motion in Experiment 1 (for a discussion of the differences, see Ratcliff et al. 2016). The flash and random dot motion tasks necessarily draw upon different perceptual processes, because the perceptual display qualitatively differs between tasks, yet this is not to say that the two tasks lead to different cognitive processes including (but not limited to) strategic adjustments to response caution.

All details of the flash task in Experiment 2 were the same as the random dot motion task in Experiment 1 with the exception of the manipulation of difficulty. There were 6 difficulty levels: $55 \%-45 \%, 60 \%-40 \%, 65 \%-35 \%, 70 \%-30 \%$, $75 \%-25 \%$, and $80 \%-20 \%$ frequency rate, where the percentage indicates the probability of a circle flashing on each frame rate of the task, and the first percentage represents the rate of one circle and the second of the other. The 6 difficulty levels

Table 1 Instructions and feedback given to participants in the test phase

\begin{tabular}{llll}
\hline Factor & Level & Instruction & Feedback \\
\hline Cue & Speed & "Focus on being as speedy as possible" & "Good time!" (if fast enough) or "Faster!" (if too slow) \\
& Accuracy & "Focus on giving as many accurate responses as possible" & "Correct!" (correct) Or "Be more accurate!" (incorrect) \\
Deadline & Deadline & "Strict deadlines will apply" & "You missed the deadline!" (no response before deadline) \\
& No deadline & "There will be no deadline for your answer" & (nil) \\
\hline
\end{tabular}


and the circle with the faster flash rate (left or right) was randomized for each trial. As in Experiment 1, the difficulty level for the test phase was selected as the smallest difference in flash rate at which a participant scored above $80 \%$ in the training session.

\section{Experiment 3}

As we show below (see Results), the deadline used in Experiment 2 might have been too slow; few responses in the deadline condition cell received "you missed the deadline!" feedback, suggesting participants did not actually experience the time pressure of a response deadline. To address this concern, in Experiment 3, we modified the flash task to include an additional earlier deadline. The experiment had a $2 \times 3$ within-subjects design with two levels for cue-based SAT and 3 levels for deadline-based SAT (early, late, and no deadline). The late deadline was set equal to the deadline in Experiment 2, namely, $1.35 \mathrm{~s}$, while the early deadline was set at $683 \mathrm{~ms}$. The early deadline was selected as the $75 \%$ percentile of the aggregate RT distribution in the deadline conditions from Experiment 2. The additional deadline achieved two goals: to set a deadline that would be early enough to induce the experience of a response deadline in the flash task but not so early as to induce chance-level accuracy and to replicate and extend the findings of Experiment 2.

Most of the presentation settings were retained from Experiment 2, with the following exceptions. The number of training trials was reduced to 30 . A lower difficulty level was selected during the training period to make the task easier since it had been found too difficult in Experiment 2. The level of difficulty selected for each participant was the hardest one at which they scored more than $90 \%$ during the training phase. The task was counterbalanced, and all participants saw all combinations of conditions. The combinatorial method used was Latin-Graeco square, because of the difficulty of presenting all possible permutations to all participants. To keep the duration of Experiment 3 similar to the duration of Experiments 1-2, the number of trials was initially set to 150 for each of the 6 blocks. After the first 5 participants had participated, it was clear that the experiment was too long. For that reason, the rest of the participants were tested for 130 trials per block (780 trials in total).

The instructions were as described in Experiment 1 and 2 with the exception of the new conditions. Unlike Experiment 1 and 2 which provided accuracy feedback only in accuracy conditions, we generalized accuracy feedback for every trial. This was done to ensure the increase of the overall level of performance. The RT comparison procedures and feedback text were as described in the previous two experiments.

\section{Results and Discussion}

We present the statistical results combined across experiments in text; E1-3 denotes Experiments 1-3. Descriptive statistics for mean RT and accuracy are shown in Table 2.

Mean RT was significantly faster in trials with deadlines than trials without deadlines $(\mathrm{E} 1-F(1,23)=22.14, p<0.001$, $\eta_{G}^{2}=0.09 ; \mathrm{E} 2-F(1,23)=16.66, p<0.001, \eta_{G}^{2}=0.12 ; \mathrm{E} 3-$ $\left.F(2,46)=72.77, p<0.001, \eta_{G}^{2}=0.43\right) .{ }^{4}$ Mean RT was also faster in speed-focused relative to accuracy-focused trials $\left(\mathrm{E} 1-F(1,23)=18.42, p<0.001, \eta_{G}^{2}=0.08 ; \mathrm{E} 2-F(1,23)=\right.$ 29.13, $p<0.001, \eta_{G}^{2}=0.19$; E3 $-F(1,23)=27.95, p<0.001$, $\left.\eta_{G}^{2}=0.13\right)$. There was a significant interaction between the cue-based and deadline-based manipulation on mean RT (E1$F(1,23)=8.89, p=0.006, \eta_{G}^{2}=0.02 ; \mathrm{E} 2-F(1,23)=13.86$, $p<0.001, \eta_{G}^{2}=0.09$; E3-F(2,46)=10.91, $p<0.001, \eta_{G}^{2}=$ $0.09)$. In each experiment, the interaction was due to responses showing greater speeding from the no-deadline to the deadline conditions in the "accuracy" regime as opposed to the "speed" regime.

Accuracy was significantly greater in trials without deadlines compared to trials with deadlines (E1$F(1,23)=14.87, p<0.001, \eta_{G}^{2}=0.04 ; \mathrm{E} 2-F(1,23)=$ $14.36, p<0.001, \eta_{G}^{2}=0.04 ; \mathrm{E} 3-F(2,46)=58.93$, $\left.p<0.001, \eta_{G}^{2}=0.30\right)$. Accuracy was also significantly greater in accuracy-focused trials compared to speedfocused trials $\left(\mathrm{E} 1-F(1,23)=18.30, p<0.001, \eta_{G}^{2}=\right.$ $0.16 ; \mathrm{E} 2-F(1,23)=41.83, p<0.001, \eta_{G}^{2}=0.20 ; \mathrm{E} 3-$ $\left.F(1,23)=20.81, p<0.001, \eta_{G}^{2}=0.08\right)$. There was a significant interaction between the cue-based and deadlinebased manipulation on accuracy in Experiments 2 and 3 $\left(\mathrm{E} 2-F(1,23)=10.54, p<0.003, \eta_{G}^{2}=0.04 ; \mathrm{E} 3-F(2,46)=\right.$ $\left.8.81, p<0.001, \eta_{G}^{2}=0.04\right)$. The nature of the interaction on choice accuracy was similar to the interaction on RT: accuracy decreased to a larger degree between the no-deadline to the deadline conditions when given accuracy-emphasis cues compared to speed-emphasis cues. The interaction was not significant in Experiment $1\left(F(1,23)=0.17, p=0.678, \eta_{G}^{2}<0.01 ; \mathrm{BF}_{10}=0.29\right)$, suggesting that the interaction between speed-accuracy trade-off manipulations on decision accuracy may be dependent on the decision task (random dot motion task vs flash task).

The analysis of mean RT and accuracy confirm that a SAT was induced in each experiment: participants were able to make faster decisions at the expense of more errors. These results were consistent across two types of perceptual decision-making task: one where evidence

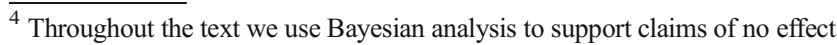
established by frequentist statistics.
} 
Table 2 Mean response time (RT) and accuracy in Experiments 1-3, including standard error of the mean (SE)

\begin{tabular}{lllll}
\hline Experiment & Cue & Deadline & Mean, ms (SE) & Accuracy (SE) \\
\hline 1 & Accuracy & No & $784(34)$ & $0.89(0.01)$ \\
& & Yes & $564(10)$ & $0.84(0.02)$ \\
& Speed & No & $571(16)$ & $0.78(0.02)$ \\
& & Yes & $494(10)$ & $0.72(0.02)$ \\
& \multirow{4}{*}{ Accuracy } & No & $1248(47)$ & $0.77(0.03)$ \\
& & Yes & $623(14)$ & $0.69(0.03)$ \\
& Speed & No & $514(15)$ & $0.64(0.03)$ \\
& & Yes & $473(11)$ & $0.63(0.03)$ \\
& Accuracy & No & $909(35)$ & $0.85(0.03)$ \\
& & Late & $613(15)$ & $0.76(0.04)$ \\
& & Early & $449(9)$ & $0.70(0.04)$ \\
& Speed & No & $647(21)$ & $0.76(0.03)$ \\
& & Late & $522(13)$ & $0.74(0.04)$ \\
& & Early & $423(9)$ & $0.68(0.04)$ \\
\hline
\end{tabular}

accumulated in discrete fashion (the flash task; Experiments 2 and 3) and another where evidence accumulated in a continuous fashion (the random-dot motion task; Experiment 1).

As expected, both deadline-based and cue-based manipulations of time-pressure induced a SAT, consistent with previous studies on time pressure. Stricter deadlines lowered choice accuracy and mean RT. Furthermore, the presence of response deadlines in the accuracy-focused conditions produced a larger SAT effect than those in speed-focused conditions, as indicated by the interaction between the two manipulations. ${ }^{5}$

The stable interaction pattern between cue-based and deadline-based manipulations of the SAT suggests that different latent components of processing may have driven the effects observed in data. In the next section we argue that cue-based manipulations affect the overall level of cautiousness (initial threshold in Fig. 1) while deadlines influence moment-to-moment adjustments to response caution. We will use the popular Diffusion Decision Model (DDM) to test this hypothesis and contrast it to alternative hypotheses.

\footnotetext{
5 Although the two SAT manipulations showed similar patterns across the two tasks, it is worth noting the discrepancies between experiments. Generally, the flash task was more difficult than the random dot motion task, as indicated by higher error rates, especially in the speed-focused trials where the presence of the deadline did not change the effect size of performance accuracy in speedfocused trials (cf. Table 2). We took this as an indication that, in Experiment 2, the feedback related to the speed cue was perceived as a deadline, potentially canceling or "blocking" the effect of the deadline manipulation. By generalizing the feedback to all conditions in Experiment 3 and reducing overall task difficulty, we observed an effect of the early deadline on the SAT in Experiment 3, consistent with the results of Experiment 1.
}

\section{Cognitive Modeling of the Differential Effects of Deadline-Based and Cue-Based SAT Manipulations}

In Experiments 1-3 we observed that the presence and the strictness of response deadlines during decision-making affect mean RT and accuracy. Though both cue-based and deadlinebased manipulations led to a trade-off between speed and accuracy, in this section, we test whether the two manipulations invoked different cognitive processes. We interpret the behavioral results in terms of a latent process of evidence accumulation by fitting fixed and collapsing threshold DDMs to the data from Experiments 1-3. We make the simplifying assumption that the cognitive processes involved in performing the random dot motion task of Experiment 1 and the flash task of Experiments 2 and 3 is approximated by the evidence accumulation process assumed in the DDM. Thus, by comparing whether the fixed or collapsing threshold models provide a more parsimonious account of each experiment, we can identify the cognitive processes that were most likely to have generated the data, given the set of models under consideration.

Based on our earlier literature review, we hypothesize that cue-based manipulations of the SAT will affect the overall amount of evidence required by the decision-makers to commit to a decision, known as boundary separation (Forstmann et al. 2008; Ratcliff and McKoon 2008; Ratcliff et al. 2016; Wagenmakers et al. 2008). In contrast, we expect that deadline-based manipulations of the SAT will cause dynamic decreases to the threshold throughout the decision process to increase the likelihood of responding before the imposed deadline, known as a collapsing threshold (Cisek et al. 2009; Evans and Hawkins 2019; Miletić and van Maanen 2019; Thura et al. 2012). This will allow us to answer the question of whether cue-based and deadline-based manipulations of the SAT are best explained by different parameters of the DDM, within the same participants in the same task.

\section{Models}

We fitted the data of each experiment with a set of models that differed in terms of which model parameters were freely estimated across conditions of the experiment and which were constrained to common values (see Table 3 ). To simplify the model comparison, we note that the mean start point bias was always set to 0 , making the assumption that on average evidence accumulation always begins equidistantly between the boundaries for the two response options (e.g., see Fig. 1 where the accumulation process starts from 0 evidence). It was not estimated since there was no explicit bias manipulation; however we do allow for random start point biases across trials (Sz, see below). 
Table 3 Description of the models fitted to Experiments $1-3$

\begin{tabular}{llll}
\hline Model & Free parameters & Constrained parameters & Fixed parameters \\
\hline f-DDM-vat-SvStSz & $v, \mathrm{~T}_{\mathrm{er}}, \alpha, \mathrm{s}_{\mathrm{v}}, \mathrm{s}_{\mathrm{t}}, \mathrm{s}_{\mathrm{z}}$ & - & $\mathrm{z}$ \\
c-DDM-vat-SvStSz & $v, \mathrm{~T}_{\mathrm{er}}, \alpha, \mathrm{s}_{\mathrm{v}}, \mathrm{s}_{\mathrm{t}}, \mathrm{s}_{\mathrm{z}}$, slope & - & $\mathrm{z}$ \\
f-DDM-vat-SvSt & $v, \mathrm{~T}_{\mathrm{er}}, \alpha, \mathrm{s}_{\mathrm{v}}, \mathrm{s}_{\mathrm{t}}$ & - & $\mathrm{z}, \mathrm{s}_{\mathrm{z}}$ \\
c-DDM-vat-SvSt & $v, \mathrm{~T}_{\mathrm{er}}, \alpha, \mathrm{s}_{\mathrm{v}}, \mathrm{s}_{\mathrm{t}}$, slope & - & $\mathrm{z}, \mathrm{s}_{\mathrm{z}}$ \\
f-DDM-vat-SvSz & $v, \mathrm{~T}_{\mathrm{er}}, \alpha, \mathrm{s}_{\mathrm{v}}, \mathrm{s}_{\mathrm{z}}$ & - & $\mathrm{z}, \mathrm{s}_{\mathrm{t}}$ \\
c-DDM-vat-SvSz & $v, \mathrm{~T}_{\mathrm{er}}, \alpha, \mathrm{s}_{\mathrm{v}}, \mathrm{s}_{\mathrm{z}}, \mathrm{slope}$ & - & $\mathrm{z}, \mathrm{s}_{\mathrm{t}}$ \\
f-DDM-vat-Sv & $v, \mathrm{~T}_{\mathrm{er}}, \alpha, \mathrm{s}_{\mathrm{v}}$ & $\mathrm{z}, \mathrm{s}_{\mathrm{z}}, \mathrm{s}_{\mathrm{t}}$ \\
c-DDM-vat-Sv & $v, \mathrm{~T}_{\mathrm{er}}, \alpha, \mathrm{s}_{\mathrm{v}}$, slope & - & $\mathrm{z}, \mathrm{s}_{\mathrm{z}}, \mathrm{s}_{\mathrm{t}}$ \\
f-DDM-a-Sv & $\alpha, \mathrm{s}_{\mathrm{v}}$ & $\mathrm{z}, \mathrm{s}_{\mathrm{z}}, \mathrm{s}_{\mathrm{t}}$ \\
c-DDM-a-Sv & $\alpha, \mathrm{s}_{\mathrm{v}}$, slope & $\mathrm{z}, \mathrm{s}_{\mathrm{z}}, \mathrm{s}_{\mathrm{t}}$ \\
c-DDM-a & $\alpha$, slope & $\gamma, \mathrm{T}_{\mathrm{er}}$ & $\mathrm{z}, \mathrm{s}_{\mathrm{z}}, \mathrm{s}_{\mathrm{t}}$ \\
\hline
\end{tabular}

Note: Free parameters were estimated independently per condition and participant. Constrained parameters were estimated per participant though held to the same value across conditions. Fixed parameters were not estimated from the data. " $v$ " stands for drift rate, " $T_{\text {er }}$ " for non-decision time, "a" for threshold, "s, for variability in drift rate, "z" for starting point, " $\mathrm{s}_{\mathrm{z}}$ " for variability in the starting point, " $\mathrm{s}_{\mathrm{t}}$ " for variability in non-decision time, and "slope" for the amount of decrease in thresholds
Table 3 shows the parameterization of all models we studied. We describe the set of models with a naming system that loosely follows Chandrasekaran and Hawkins (2019). The principle of the naming system is that a model is augmented with a character for each additional parameter freely estimated across conditions; the characters for each parameter are shown in the notes to Table 3. Parameters that are freely estimated from data though constrained to the same value across conditions are not referred to in the model name. For illustration, the model in the upper row of Table 3 is named f-DDM-vatSvStSz because it is a fixed thresholds DDM (f-DDM) that freely estimates the base parameters of drift rate (v), threshold (a) and non-decision time (t), and across-trial variability parameters for drift rate $(\mathrm{Sv})$, non-decision time $(\mathrm{St})$, and starting point (Sz). By comparison, the model in the final row of Table 3 is named c-DDM-a because it is a collapsing threshold DDM (c-DDM) that freely estimates the threshold (a) and the slope of the collapsing threshold (which is encapsulated in the "c" of "c-DDM").

The number of freely estimated parameters in each model for each participant is determined by multiplying the number of parameters listed in the "free parameters" column of Table 3 by the number of conditions in the experiment ( 4 in Experiments 1 and 2, 6 in Experiment 3) plus the number of parameters listed in the "constrained parameters" column. For example, in model f-DDM-vat-Sv, there are 4 (parameters) $\times 4$ $(\mathrm{E} 1 / \mathrm{E} 2)$ or $6(\mathrm{E} 3)+0=16(\mathrm{E} 1 / \mathrm{E} 2)$ or $24(\mathrm{E} 3)$ parameters per participant. For c-DDM-vat-Sv, the collapsing thresholds equivalent, there are $20(\mathrm{E} 1 / 2)$ or 30 (E3) parameters per participant.

Some model parameters were of particular relevance to our hypotheses. In the collapsing threshold DDMs, the threshold parameter indicates the initial level of response caution (boundary separation at time 0 ), and the slope indicates how quickly this caution changes as the time since stimulus onset increases (rate of decline). We expect that the cue-based manipulation but not deadline-based manipulation will influence the initial threshold, and we expect that the deadline-based manipulation but not the cue-based manipulation will influence the slope. Therefore, if both hypotheses are supported, we expect evidence for a model with collapsing thresholds (any of the c-DDM models). For simplicity, we assume all collapsing thresholds decrease linearly as a function of elapsed decision time, which is a form of collapsing thresholds that is well-identified in data (Evans et al. 2019). For the fixed threshold DDMs, the threshold parameter indicates the general response caution of the participant, which is independent of elapsed decision time. Therefore, if there is support for our hypothesis about cue-based manipulations but not deadlinebased manipulations, we expect evidence for a model with fixed thresholds (any of the f-DDM models).

We also considered the across-trial variability parameters of the DDM. Across-trial variability in drift rate was of particular importance as it plays an important role in allowing a fixed-threshold DDM to capture slow errors (Ratcliff 1978) which are also predicted by collapsing thresholds without across-trial variability in the drift rate (Hawkins et al. 2015b). As a result, by estimating drift rate variability, we create a level playing field in terms of the capacity for collapsing and fixed threshold DDMs to account for slow errors, which were present in the data. If across-trial variability in drift rate is the cause of slow errors, then we expect evidence for any of the f-DDM models with Sv, though if slow errors are instead due to collapsing thresholds, we expect support for any of the c-DDM models, which may also have an additional contribution from variability in drift rate. We also tested 
whether the additional flexibility of across-trial variability in starting point and/or non-decision time accounted for unique variance in the data not already explained by fixed or collapsing thresholds with or without drift rate variability. If this additional flexibility is required to account for the data, then we expect to support models with St and/or St. ${ }^{6}$ We also tested much more constrained models by enforcing the same drift rate and non-decision time across conditions (f-DDM-a-Sv, cDDM-a-Sv). Comparison of f-DDM-a-Sv with c-DDM-a (without drift rate variability) allowed us to test the unique contribution of the drift rate variability parameter and its potential substitutability with the collapsing threshold parameter.

\section{Fitting Procedure}

We followed the parameter estimation routines of Hawkins et al. (2015a). We used Monte Carlo simulation with 10,000 samples per experimental condition during parameter estimation, which was conducted by quantile maximum probability estimation. To ensure the comparison between the models was fair, we used the same simulation-based method for parameter fitting of the DDM with fixed thresholds. We set the diffusion coefficient $(\mathrm{s}=.1)$ as a scaling parameter and fitted the DDM to RT in seconds. In addition, we verified that the fixed and collapsing threshold models recovered almost all of the estimated parameters well (yet, the drift rate variability parameter recovered less well than other parameters). For details of the parameter recovery, see Supplementary Materials, and for a more complete treatment of parameter recovery in collapsing threshold models, see Evans et al. (2019).

The best-fitting parameters for all models listed in Table 3 were independently estimated for each participant in each experiment, which makes the model application consistent with the within-subject design of the three experiments. Parameters were estimated through the Differential Evolution optimization algorithm (Ardia et al., 2011) with a maximum of 1000 iterations.

\section{Model Comparison and Model Fit}

To assess which models provided the most parsimonious account of the data - that is, the best balance between goodnessof-fit to data and model flexibility - we used the Akaike Information Criterion (AIC), along with its correction for small samples (AICc), and the Bayesian Information Criterion (BIC). These three metrics are used as a standard practice in model comparison (Akaike 1974; Schwarz 1978; see also Heathcote et al. 2015). The three measures are based on the quantile maximum log-likelihood of the fitted models

\footnotetext{
${ }^{6}$ When interpreting these results, the reader should take into account that estimation of across-trial variability parameters can be challenging in some contexts (see for example: Boehm et al. 2018).
}

and reward goodness-of-fit but penalize for extra free parameters, with different complexity penalties across the three metrics. Lower values indicate more parsimonious accounts of data than higher values. ${ }^{7}$

Table 4 shows the model comparison outcomes. All three experiments provided the most support for the same model: collapsing thresholds with across-trial variability in drift rate (c-DDM-vat-Sv). For models that did not include across-trial variability in starting point and non-decision time, the collapsing thresholds version of each model tended to provide the best explanation of the data, though not in all cases. However, for models that did include those sources of additional variability, the fixed thresholds models tended to outperform the collapsing thresholds models, though again not in all cases. Nevertheless, there was no combination of the variability parameters in the fixed thresholds model that provided a better explanation of the data than the best collapsing thresholds model. Generally, the models that allowed drift rates and non-decision times to vary across conditions showed a much better performance than those in which these parameters were constrained to the same value across conditions, in spite of the increased number of free parameters. Interestingly, omitting drift rate variability in c-DDM-a substantially worsened performance of the collapsing model, which underscores the fact that drift rate variability makes a unique contribution to the model and cannot be substituted by the slope parameter. ${ }^{8}$ For the remainder of the results, we explore the performance of the best performing collapsing threshold model (c-DDM-vat-Sv) and best performing fixed threshold model (f-DDM-vat-Sv). For simplicity, we refer to these models simply as the collapsing and fixed threshold models rather than repeatedly naming their precise parameterizations.

To provide absolute model fit diagnostics, the fit of the preferred collapsing threshold model is shown in Figs. 2 and 3, taking Experiment 2 as an example. The model provides a very good account of the data, with correlations between observed and predicted data (RT quantiles and accuracy) greater than 0.97. The fit of the same model to Experiments 1 and 3 was equally good (see Supplementary Materials: Figs. 3-6). In Experiment 1, the model fit strongly correlated with observed data in correct responses (above 0.97), but error responses exhibited higher variability, especially in the conditions without a deadline (correlations above 0.70). Higher variability in fitting error responses is expected because of the fewer errors made in no-deadline conditions; a

\footnotetext{
$\overline{7}$ We arrived at the same conclusions by performing chi-square tests on model deviances based on average log-likelihoods.

${ }^{8}$ Another way to establish the unique contribution of the slope parameter is to look at correlations among the estimated model parameters. For example, in Experiment 3, the slope parameter did not correlate with the drift rate variability parameter; a finding which points to the unique contribution of each of these two parameters in explaining the data (see Supplementary Materials: Figure 15 and Table 1).
} 
Table 4 Model comparison between fixed and collapsing threshold DDMs

\begin{tabular}{|c|c|c|c|c|c|}
\hline Experiment & Model & \# of parameters & AIC & $\mathrm{AICc}$ & $\mathrm{BIC}$ \\
\hline \multirow[t]{11}{*}{ Experiment 1} & f-DDM-vat-SvStSz & $576(24)$ & 86,179 & 86,215 & 90,704 \\
\hline & c-DDM-vat-SvStSz & $672(28)$ & 87,075 & 87,124 & 92,354 \\
\hline & f-DDM-vat-SvSt & $480(20)$ & 85,905 & 85,930 & 89,676 \\
\hline & c-DDM-vat-SvSt & $576(24)$ & 87,092 & 87,128 & 91,617 \\
\hline & f-DDM-vat-SvSz & $480(20)$ & 86,912 & 86,937 & 90,683 \\
\hline & c-DDM-vat-SvSz & $576(24)$ & 87,035 & 87,071 & 91,560 \\
\hline & f-DDM-vat-Sv & $384(16)$ & 87,028 & 87,045 & 90,045 \\
\hline & c-DDM-vat-Sv & $480(20)$ & 85,805 & 85,831 & 89,577 \\
\hline & f-DDM-a-Sv & $240(10)$ & 343,907 & 343,911 & 345,869 \\
\hline & c-DDM-a-Sv & $336(14)$ & 161,917 & 161,929 & 164,557 \\
\hline & c-DDM-a & $264(11)_{-}$ & 457,219 & 457,177 & 459,293 \\
\hline \multirow[t]{11}{*}{ Experiment 2} & f-DDM-vat-SvStSz & $576(24)$ & 92,089 & 92,125 & 96,613 \\
\hline & c-DDM-vat-SvStSz & $672(28)$ & 92,520 & 92,569 & 97,797 \\
\hline & f-DDM-vat-SvSt & $480(20)$ & 91,991 & 92,016 & 95,761 \\
\hline & c-DDM-vat-SvSt & $576(24)$ & 92,349 & 92,385 & 96,873 \\
\hline & f-DDM-vat-SvSz & $480(20)$ & 92,658 & 92,683 & 96,428 \\
\hline & c-DDM-vat-SvSz & $576(24)$ & 92,309 & 92,345 & 96,833 \\
\hline & f-DDM-vat-Sv & $384(16)$ & 92,417 & 92,433 & 95,433 \\
\hline & c-DDM-vat-Sv & $480(20)$ & 91,661 & 91,685 & 95,430 \\
\hline & f-DDM-a-Sv & $240(10)$ & 228,937 & 228,943 & 230,822 \\
\hline & c-DDM-a-Sv & $336(14)$ & 204,389 & 204,401 & 207,028 \\
\hline & c-DDM-a & $264(11)$ & 477,437 & 477,445 & 479,511 \\
\hline \multirow[t]{11}{*}{ Experiment 3} & f-DDM-vat-SvStSz & $864(36)$ & 88,386 & 88,471 & 95,143 \\
\hline & c-DDM-vat-SvStSz & $1008(42)$ & 89,081 & 89,198 & 96,964 \\
\hline & f-DDM-vat-SvSt & $720(30)$ & 88,125 & 88,184 & 93,755 \\
\hline & c-DDM-vat-SvSt & $864(36)$ & 88,801 & 88,886 & 95,557 \\
\hline & f-DDM-vat-SvSz & $720(30)$ & 89,538 & 89,597 & 95,169 \\
\hline & c-DDM-vat-SvSz & $864(36)$ & 88,885 & 88,971 & 95,642 \\
\hline & f-DDM-vat-Sv & $576(24)$ & 89,147 & 89,184 & 93,651 \\
\hline & c-DDM-vat-Sv & $720(30)$ & 87,800 & 87,859 & 93,431 \\
\hline & f-DDM-a-Sv & $336(14)$ & 148,968 & 148,981 & 151,596 \\
\hline & c-DDM-a-Sv & $480(20)$ & 134,873 & 134,899 & 138,627 \\
\hline & c-DDM-a & $360(15)$ & 490,338 & 490,338 & 493,153 \\
\hline
\end{tabular}

Note: Models that are preferred according to each model selection metric are presented in bold per Experiment. The number of parameters refers to the total number estimated across all participants (in parentheses, the number of estimated parameters per participant is also given). Raw AIC, AICc, and BIC values are given rounded to nearest whole number phenomenon observed in model fits of other tasks as well (Palminteri et al. 2017). In Experiment 3, the collapsing threshold model slightly underestimated accuracy in the early deadline condition though all correlations between observed and predicted values were above 0.95 for both error and correct responses.

To understand why the collapsing threshold model was preferred over its nearest fixed thresholds competitor (fDDM-vat-Sv), we explored which quantitative patterns in data the fixed thresholds model missed. The goodness of fit to data of the fixed threshold model had some similar strengths and weaknesses to the collapsing threshold model: higher variability in error responses and underestimation of the observed accuracy in the early deadline condition of Experiment 3 (see Figs. 7-12 of Supplementary Materials). However, the fixed threshold model also tended to underestimate the RT distributions in all quantiles, especially in Experiments 1 and 3. This result is shown more completely in Fig. 4. The fixed threshold model underestimates the central body of the RT distributions ( 0.3 and 0.5 quantiles) but heavily overestimates the tails ( 0.9 quantile 0.9 ). This happens because the fixed threshold model predicts heavier-tailed distributions than what was observed in data. To predict shorter-tailed distributions, the fixed thresholds model underpredicts the main body of the 
Fig. 2 Fit of model c-DDM-vatSv to the correct responses of Experiment 2. Observed vs. predicted accuracy and quantile RTs per participant and condition are shown. RTs are given for the 0.1 , $0.3,0.5,0.7$, and 0.9 quantiles. Lines represent the identity function
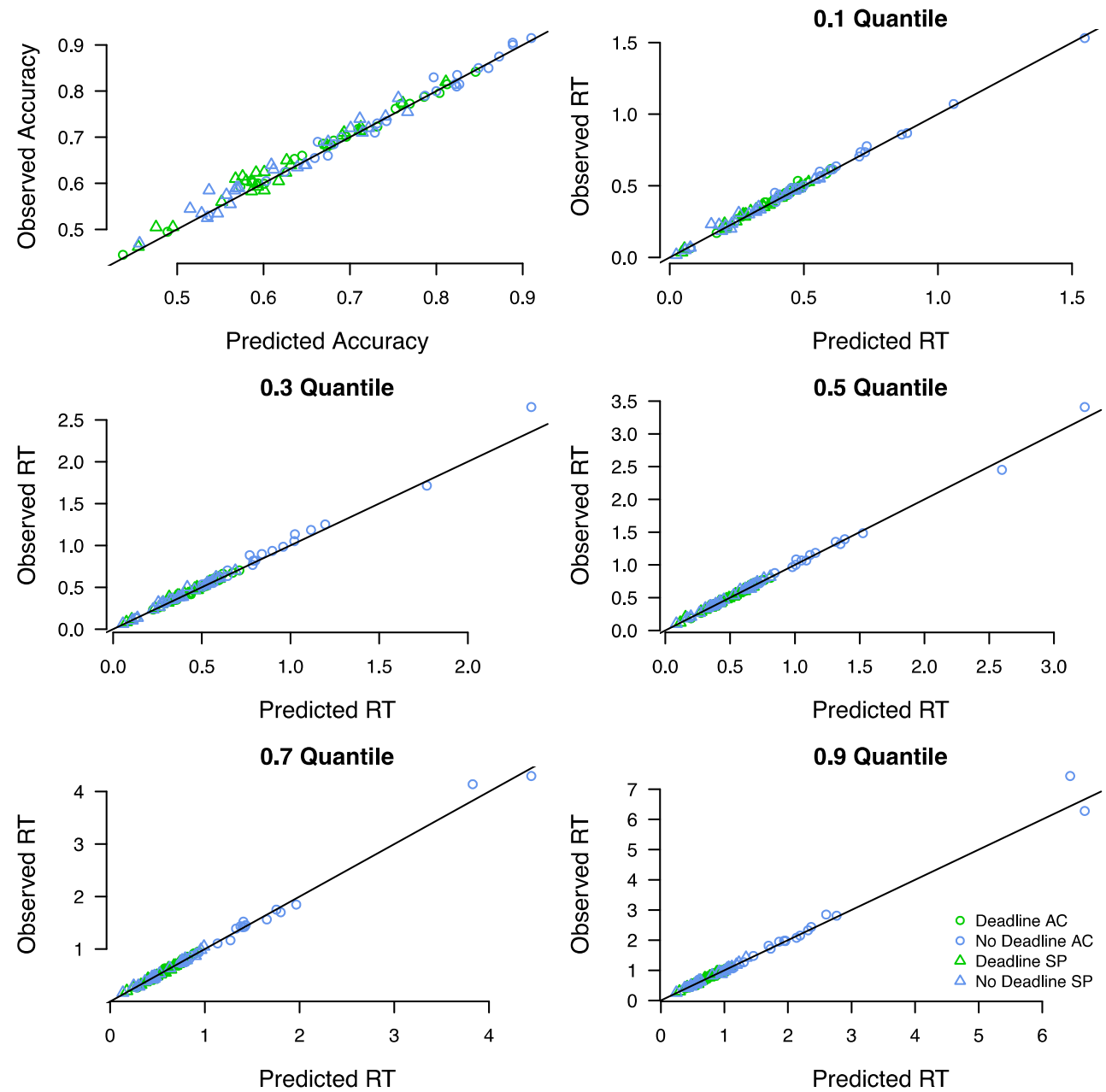

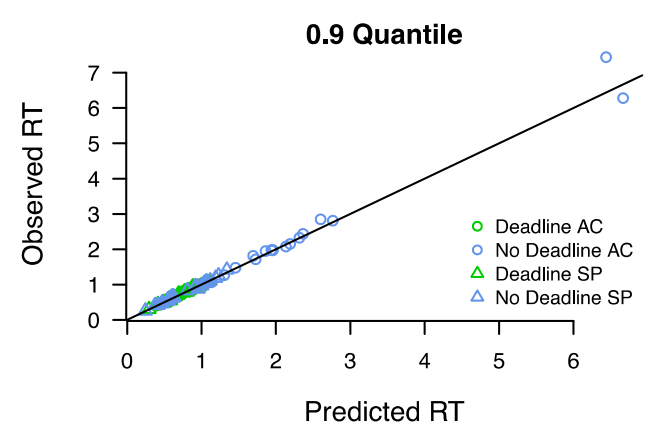

RT distributions. The collapsing threshold model appears to fit the data better. Similar patterns were observed in the other two experiments; see Supplementary Materials Figs. 13-14.

\section{Model Parameters}

We now evaluate how the parameters of the best model of the data (i.e., the collapsing threshold model) changed across the conditions of the three Experiments. Figures 5 and 6 provide a graphical presentation of the model parameters. Figure 5 presents the model parameters of greatest interest to our hypotheses: the initial value of the response threshold and the rate of decline in that threshold as the time from stimulus onset increases. Figure 6 presents the rest of the parameters: drift rate, drift rate variability, and non-decision time.

Across all experiments, the response thresholds declined to a significantly greater extent in trials with deadlines than trials without deadlines $\left(\mathrm{E} 1-F(1,23)=9.97, p=0.004, \eta_{G}^{2}=0.05\right.$; $\mathrm{E} 2-F(1,23)=5.20, p=0.032, \eta_{G}^{2}=0.04$; E3 $-F(1,46)=$ $\left.58.75, p<0.001, \eta_{G}^{2}=0.45\right)$. In contrast, the thresholds declined at different rates for speed-focused relative to accuracy- focused trials only in Experiment $2(F(1,23)=5.75, p=0.024$, $\left.\eta_{G}^{2}=0.04\right)$, not Experiments 1 or $3(\mathrm{E} 1-F(1,23)=1.33, p=$ $0.260, \eta_{G}^{2}=0.01 ; \mathrm{BF}_{10}=0.43 ; \mathrm{E} 3-F(1,23)=0.13, p=0.718$, $\left.\eta_{G}^{2}<0.01 ; \mathrm{BF}_{10}=0.18\right)$. There was also no interaction between the cue-based and deadline-based manipulation on the slope parameter in Experiments 1 and $3(\mathrm{E} 1-F(1,23)=3.97$, $p=0.058, \eta_{G}^{2}=0.02 ; \mathrm{BF}_{10}=0.99 ; \mathrm{E} 3-F(1,46)=1.10, p=$ $\left.0.339, \eta_{G}^{2}=0.01 ; \mathrm{BF}_{10}=0.24\right)$, though there was a significant interaction in Experiment $2\left(F(1,23)=6.19, p=0.020, \eta_{G}^{2}=\right.$ 0.03 ). As seen in Fig. 5, this interaction was driven by the shallow decline in the threshold in the accuracy - nodeadline condition compared to the steeper decline in the remaining three conditions. It is worth noting that a similar trend was present in Experiment 1 though the pattern was not statistically significant.

In contrast, the initial value of the threshold - an indicator of the overall level of response caution - was significantly greater in accuracy-focused compared to speed-focused trials across all three experiments $(\mathrm{E} 1-F(1,23)=5.63, p=0.026$, $\eta_{G}^{2}=0.01 ; \mathrm{E} 2-F(1,23)=12.16, p=0.001, \eta_{G}^{2}=0.10 ; \mathrm{E} 3-$ $\left.F(1,23)=12.65, p=0.001, \eta_{G}^{2}=0.07\right)$. The initial threshold 
Fig. 3 Fit of model c-DDM-vat$\mathrm{Sv}$ to the error responses of Experiment 2. Observed vs. predicted quantile RTs per participant and condition are shown. RTs are given for the $0.1,0.3,0.5,0.7$, and 0.9 quantiles. Lines represent the identity function
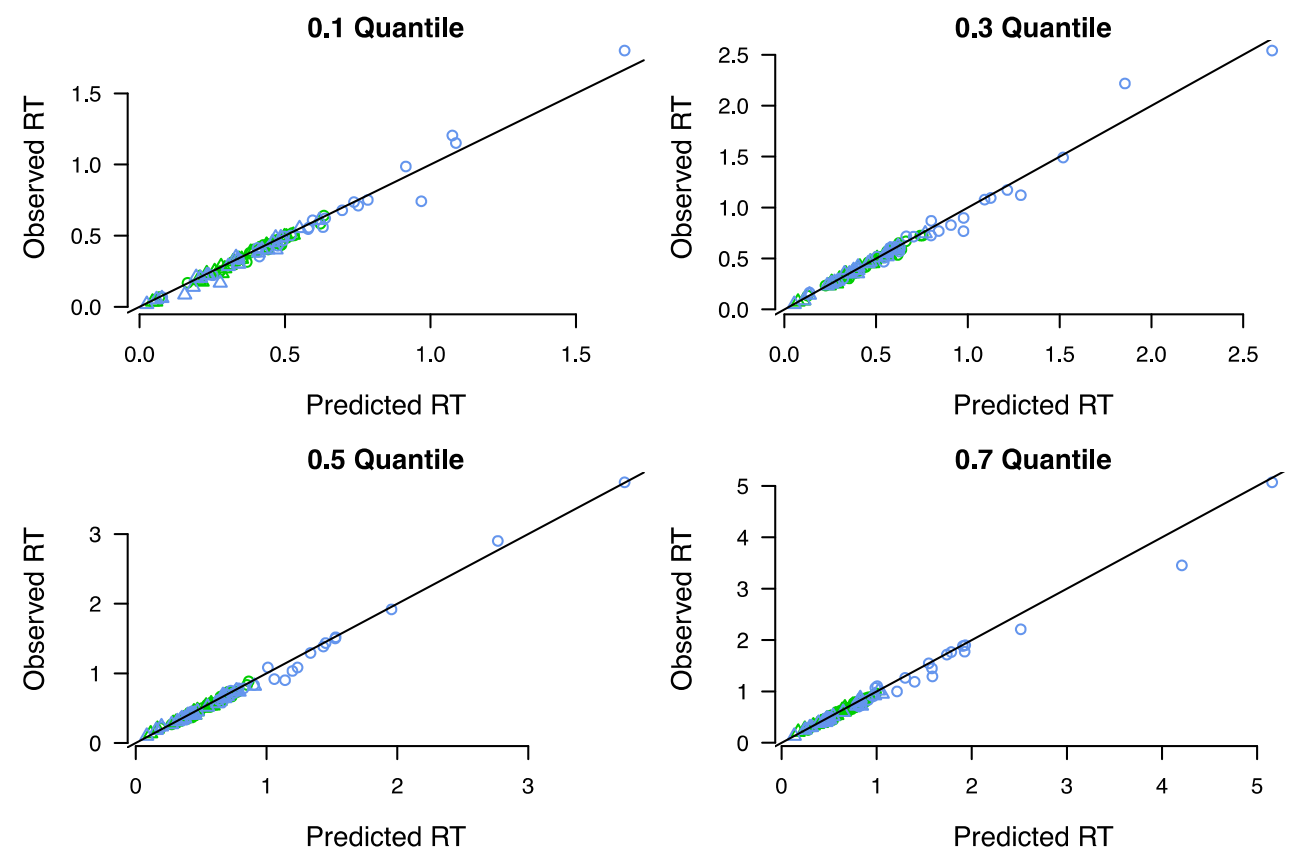

0.9 Quantile

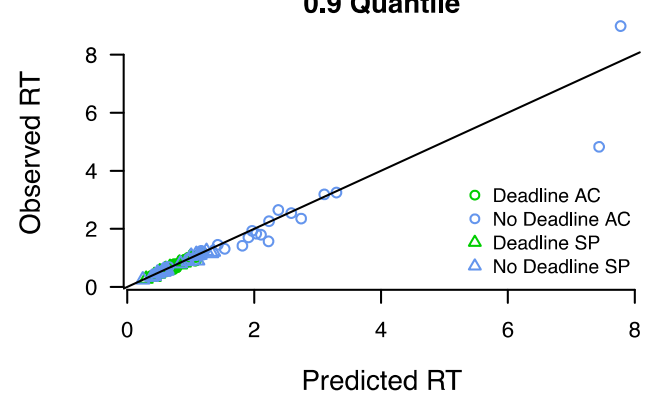

Predicted RT was also greater in trials without a deadline compared to with a deadline in Experiments 1 and $2(\mathrm{E} 1-F(1,23)=7.27, p=$ $0.012, \eta_{G}^{2}=0.02$; E2 $\left.-F(1,23)=6.60, p=0.017, \eta_{G}^{2}=0.05\right)$, but not Experiment $3\left(F(1.46)=2.61, p=0.084, \eta_{G}^{2}=0.03\right.$; $\left.\mathrm{BF}_{10}=0.65\right)$. The interaction followed a similar pattern; not significant in Experiments 1 and $2(\mathrm{E} 1-(1,23)=0.34, p=$ $0.563, \eta_{G}^{2}<0.01 ; \mathrm{BF}_{10}=0.30 ; \mathrm{E} 2-F(1,23)=3.38, p=$ $\left.0.078, \eta_{G}^{2}=0.02 ; \mathrm{BF}_{10}=0.92\right)$ though significant in Experiment $3\left(F(1,46)=5.78, p=0.005, \eta_{G}^{2}=0.06\right)$. The interaction was driven by the early deadline - speed condition, which showed higher levels of threshold than expected. This was likely because this condition had a very large slope relative to the other deadline conditions, so to compensate the initial threshold was set to a higher value.

For the rest of the model parameters (Fig. 6), we observed that the parameter of non-decision time was slower in the accuracy-focused trials than in speed-focused ones in Experiments 1 and 2 but not in Experiment $3(F(1,23)=$ $11.41, p=0.002, \eta_{G}^{2}=0.07$; E2 $-F(1,23)=10.05, p=0.004$, $\eta_{G}^{2}=0.07$; E3-F $(1,23)=2.66, p=0.116, \eta_{G}^{2}=0.02 ; \mathrm{BF}_{10}=$ 0.60 ). In addition, non-decision time was faster in deadline conditions but only in Experiment 3 while the rest of the Experiments did not show significant differences (E1$F(1,23)=2.14, p=0.156, \eta_{G}^{2}<0.01 ; \mathrm{BF}_{10}=0.25$; E2$F(1,23)=0.46, p=0.502, \eta_{G}^{2}<0.01$; E3 $-F(1,46)=25.85$, $p<0.001, \eta_{G}^{2}=0.16$ ). No interaction was observed (E1$F(1,23)=0.13, p=0.719, \eta_{G}^{2}<0.01 ; \mathrm{BF}_{10}=0.30 ; \mathrm{E} 2-$ $F(1,23)=1.25, p=0.274, \eta_{G}^{2}<0.01 ; \mathrm{BF}_{10}=0.31$; E3$\left.F(1,46)=0.38, p=0.680, \eta_{G}^{2}<0.01 ; \mathrm{BF}_{10}=0.14\right)$.

The drift rate parameter - the rate perceptual information is accumulated by the decision-maker - showed a clear pattern according to which it was higher in across accuracy-focused than speed-focused trials $\left(\mathrm{E} 1-F(1,23)=20.85, p<0.001, \eta_{G}^{2}\right.$ $=0.12$; E2 $-F(1,23)=14.34, p<0.001, \eta_{G}^{2}=0.07$; E3$\left.F(1,23)=4.28, p=0.049, \eta_{G}^{2}=0.01\right)$ as well as in trials without deadlines than in trials with deadlines in two out of the three Experiments $\left(\mathrm{E} 1-F(1,23)=10.67, p=0.003, \eta_{G}^{2}=0.03\right.$; $\mathrm{E} 2-F(1,23)=0.02, p=0.870, \eta_{G}^{2}<0.01 ; \mathrm{BF}_{10}=0.21$; E3$\left.F(1,46)=15.53, p<0.001, \eta_{G}^{2}=0.12\right)$. Additionally, no interaction was observed between these two factors $(\mathrm{E} 1-F(1,23)=$ $0.15, p=0.700, \eta_{G}^{2}<0.01 ; \mathrm{BF}_{10}=0.29 ; \mathrm{E} 2-F(1,23)<0.001$, 
Fig. 4 Deviation between observed and predicted RTs for the $0.1,0.3,0.5,0.7$, and 0.9 quantiles of the RT distribution separately for each condition in Experiment 2, averaged across participants. Continuous lines indicate the fixed threshold model, and dashed lines indicate the collapsing threshold model. Bars represent standard errors
AC - Deadline

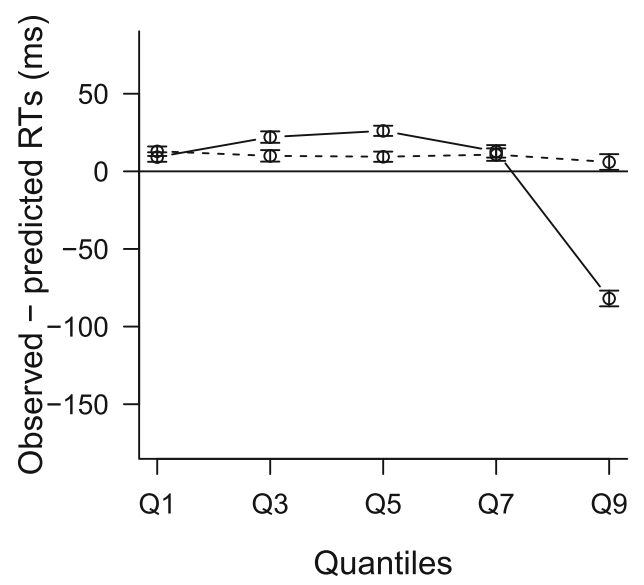

tiles

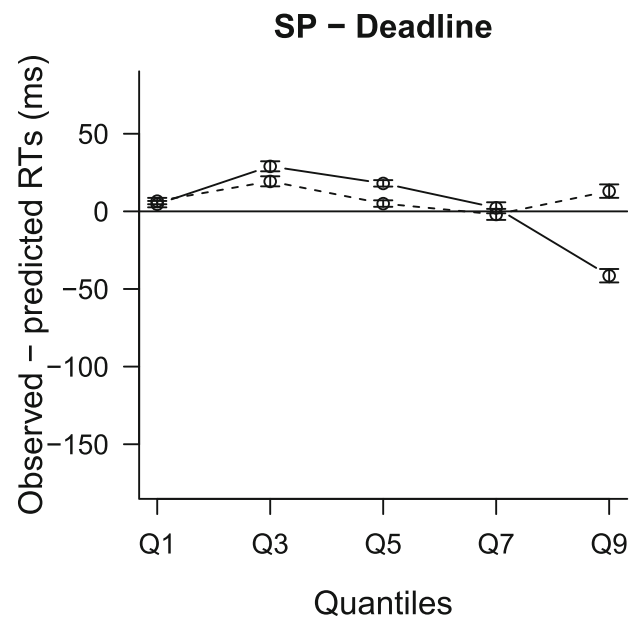

AC - No Deadline

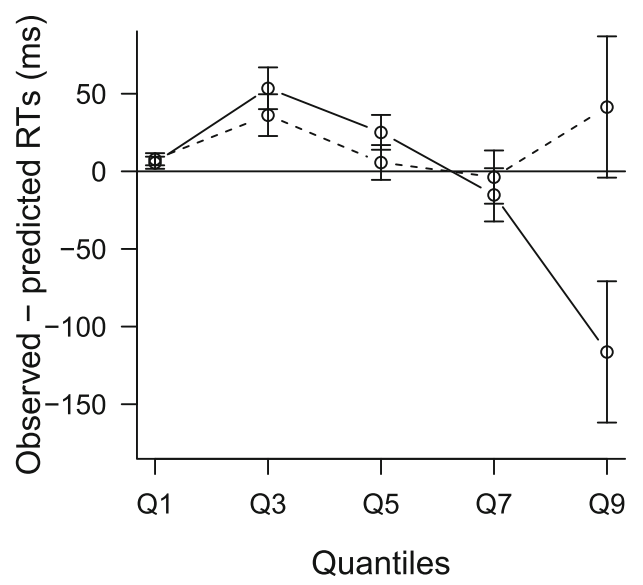

SP - No Deadline

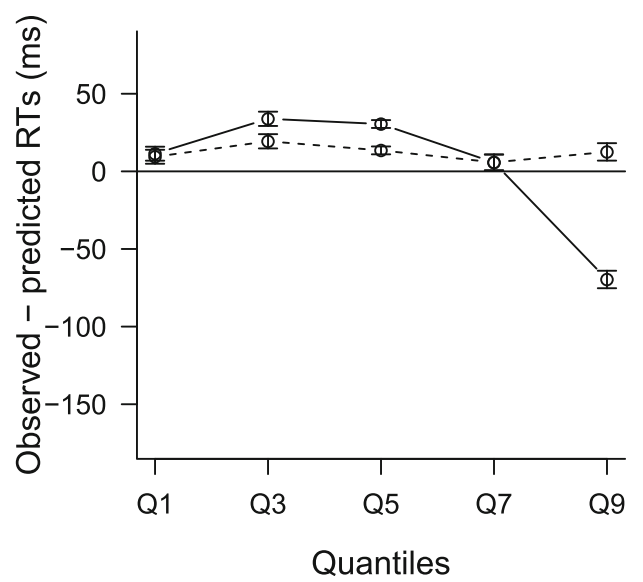

$p=0.992, \eta_{G}^{2}<0.01 ; \mathrm{BF}_{10}=0.29 ; \mathrm{E} 3-F(1,46)=0.37, p=$ $\left.0.691, \eta_{G}^{2}<0.01 ; \mathrm{BF}_{10}=0.13\right)$.

On the other hand, the drift rate variability parameter showed mixed results (for a relationship between the drift rate and drift rate variability see Fig. 6: last right column); it was higher in conditions without deadlines in Experiment 1, but it was lower for deadline conditions in Experiment 2, while no significant difference was found for Experiment 3 (E1$F(1,23)=5.27, p=0.031, \eta_{G}^{2}=0.13 ; \mathrm{E} 2-F(1,23)=5.35$, $p=0.029, \eta_{G}^{2}<0.02 ; \mathrm{E} 3-F(1,46)=0.16, p=0.850, \eta_{G}^{2}<$ $0.01 ; \mathrm{BF}_{10}=0.13$ ). Furthermore, that model parameter did not significantly change across speed-focused vs. accuracyfocused trials $\left(\mathrm{E} 1-F(1,23)=0.03, p=0.866, \eta_{G}^{2}<0.01\right.$; $\mathrm{BF}_{10}=0.21 ; \mathrm{E} 2-F(1,23)=2.17, p=0.154, \eta_{G}^{2}<0.01$; $\mathrm{BF}_{10}=0.49 ; \mathrm{E} 3-F(1,23)=1.94, p=0.176, \eta_{G}^{2}=0.01$; $\left.\mathrm{BF}_{10}=0.51\right)$. No interaction between the two factors was observed in the drift rate variability parameter $(\mathrm{E} 1-F(1,23)=$ $0.03, p=0.856, \eta_{G}^{2}<0.01 ; \mathrm{BF}_{10}=0.29 ; \mathrm{E} 2-F(1,23)=0.11$, $p=0.734, \eta_{G}^{2}<0.01 ; \mathrm{BF}_{10}=0.30 ; \mathrm{E} 3-F(1,46)=1.94, p=$ $0.176, \eta_{G}^{2}=0.01 ; \mathrm{BF}_{10}=0.26$ ).

Taken together, the deadline-based manipulations were most consistently associated with the slope parameter of the collapsing threshold model such that response deadlines induced steeper slopes than no-deadline conditions in Experiments 1 and 2, with the same pattern observed across the three deadline levels in Experiment 3. The threshold parameter, on the other hand, was most consistently associated with the cue-based manipulation such that speed-focused trials reduced the response threshold relative to accuracy-focused trials. This result aligns with a large body of literature (Evans et al. in press; Forstmann et al. 2008; Frazier and Yu 2007; Hawkins et al. 2015b; Rae et al. 2014; Ratcliff and McKoon 2008; Van Maanen et al. 2011; Wagenmakers et al. 2008). In addition, trials with deadlines and with speedfocused instructions produced lower drift rates than trials without deadlines or with accuracy-focused instructions. However, we found mixed results for the association of the non-decision time parameter with deadline conditions (the non-decision time parameter was faster in conditions with deadlines only in Experiment 3 in line with previous studies, e.g., Murphy et al. 2016) or the association of the drift rate variability with deadline conditions (drift rate variability was higher for trials with deadlines only in Experiment 2). 

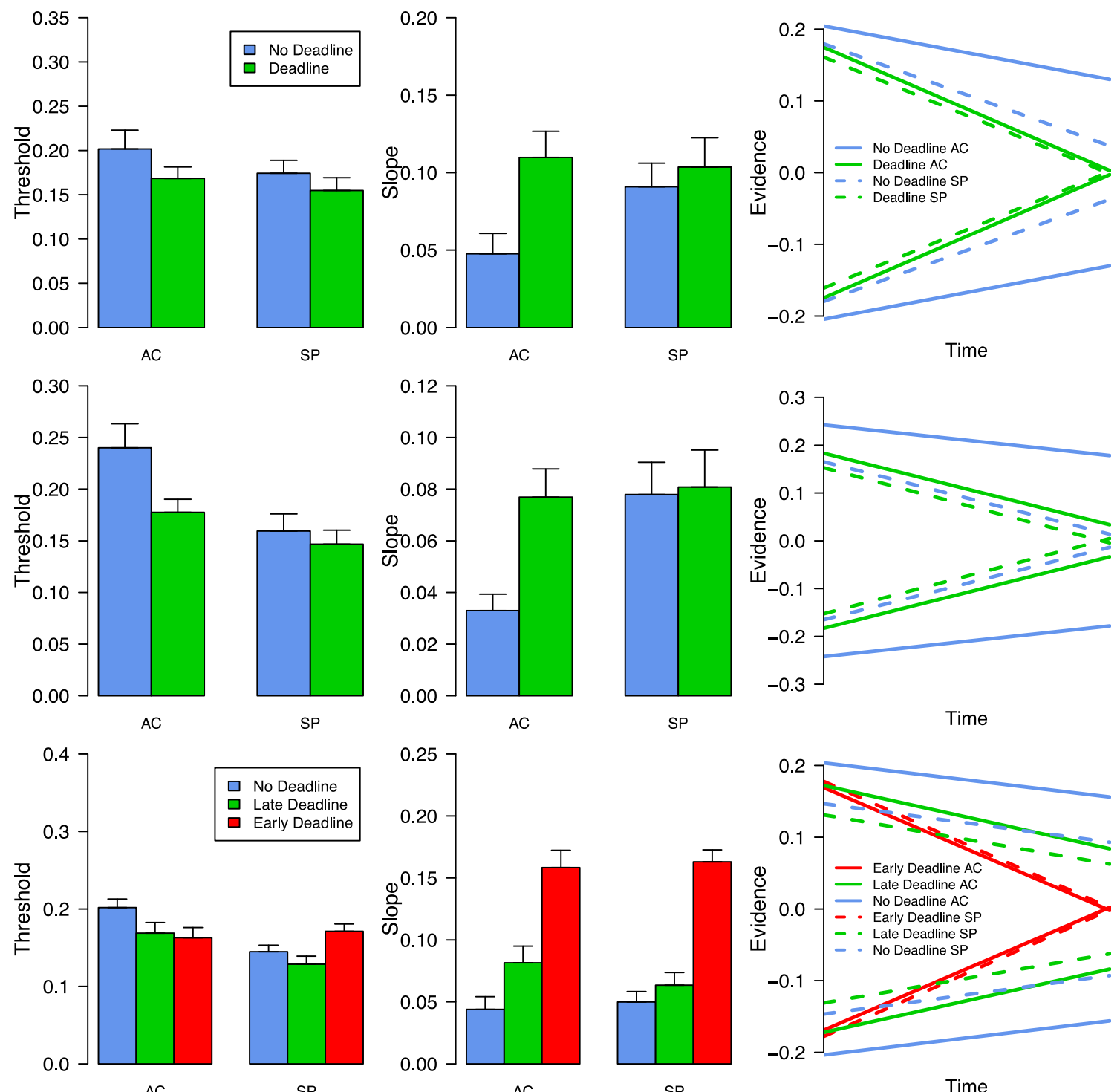

$\mathrm{AC}$

$\mathrm{SP}$

Time

Fig. 5 Mean slope and threshold parameters of the collapsing thresholds model. Top row: Experiment 1. Middle row: Experiment 2. Bottom row: Experiment 3. Means are given with standard errors. "AC" and "SP"

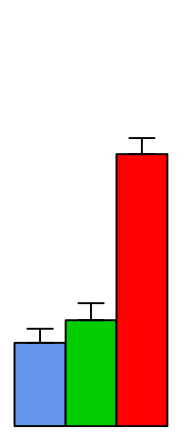

SP

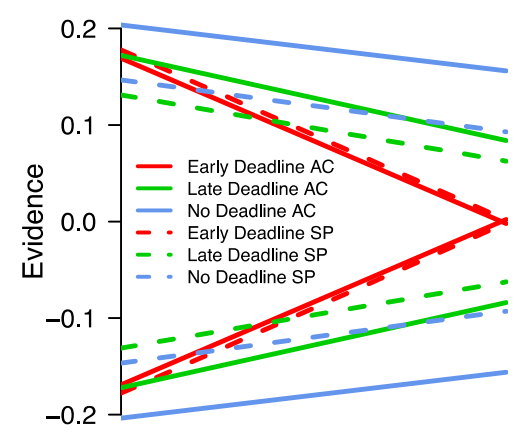

Time

stands for "accuracy" and "speed" respectively. The right-hand column provides a mean representation of the threshold and slope parameters

\section{General Discussion}

We have provided evidence that deadline-based and cuebased manipulations of the speed-accuracy trade-off have different psychological effects on latent decision-making processes. Our results indicate that cue-based manipulations affect the general level of response caution with which people make perceptual decisions. Throughout the course of a decision, this overall level is subject to dynamic changes due to a potential pressure to respond. Such time pressure can be experimentally induced through deadlines but is less sensitive to pre-stimulus cues. These results are in line with previous empirical investigations of deadline and instruction effects on human RT and accuracy data (Forstmann et al. 2010, 2008; Frazier and Yu 2007; Karşılar et al. 2014; Miletić and van
Maanen 2019; Pike 1968; Pike and Dalgleish 1982; Van Maanen et al. 2011; Van Zandt et al. 2000).

The differences between the overall level of caution and how it changes throughout the course of a decision are wellexplained within the theoretical framework of evidence accumulation models (such as the DDM) that incorporate collapsing thresholds. We found that the best explanation of the data assigned the effect of a cue-based manipulation of the SAT to the overall level of the decision threshold and the effect of a deadline-based manipulation of the SAT to dynamically collapsing thresholds. The model also assumed that mean drift rate, drift rate variability, and non-decision time changed across conditions. This model is preferable for three reasons. First, it provides a coherent conceptual account of the cognitive mechanisms triggered by time pressure manipulations 

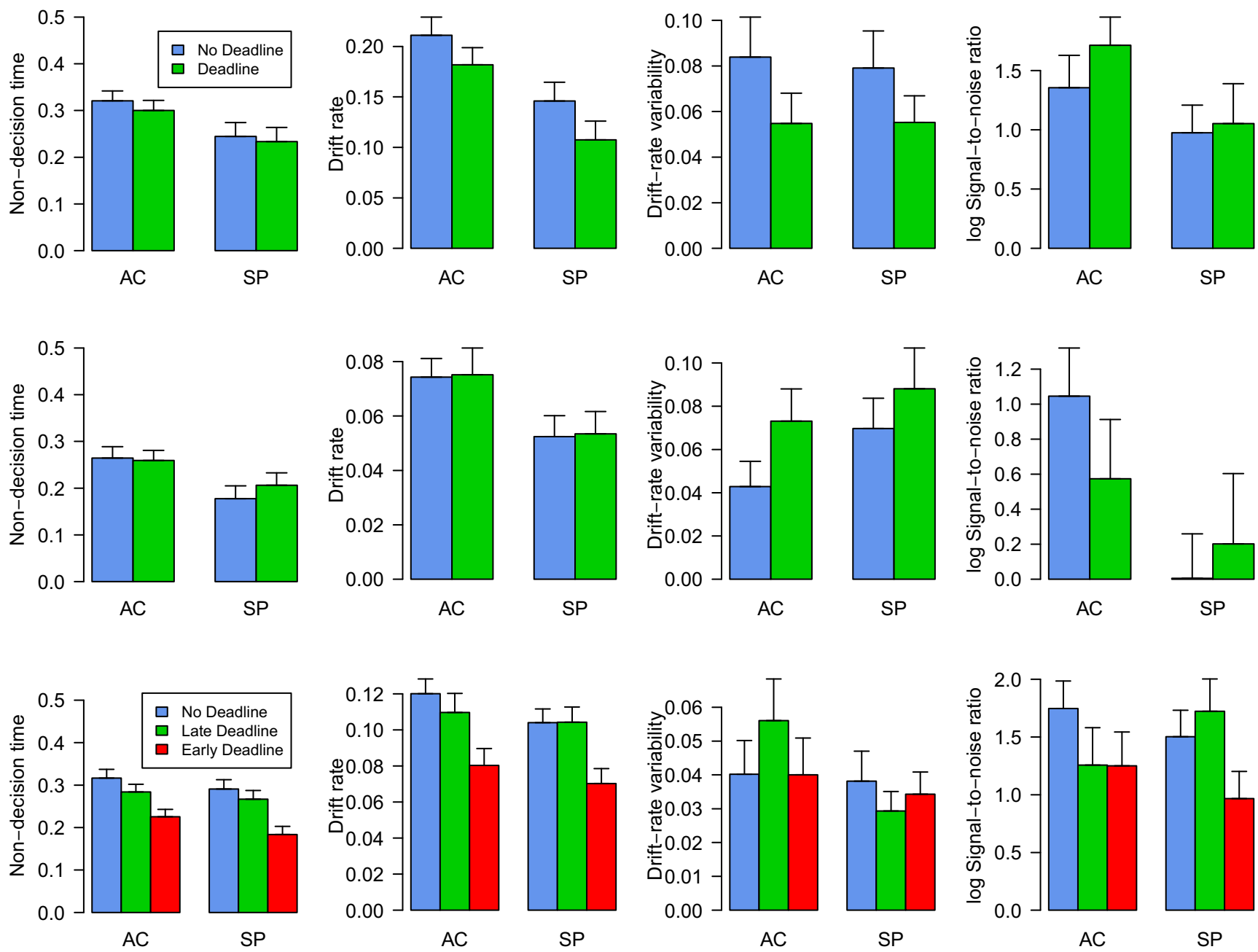

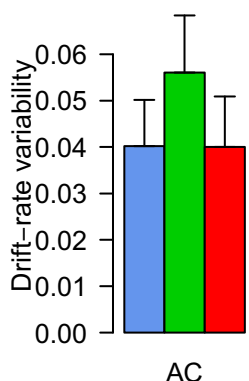

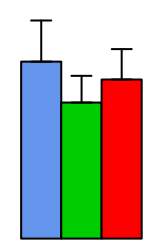

SP

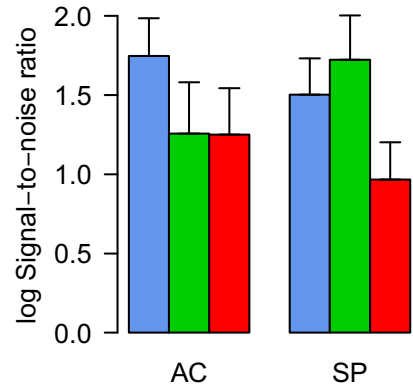

Experiment 1. Middle row: Experiment 2. Bottom row: Experiment 3. Means are given with standard errors. "AC" and "SP" stands for "accuracy" and "speed" respectively

Supplementary mMterials). A change in the proportion of guess responses due to a deadline therefore does not seem likely. For this reason, we did not explore this class of models further. Nevertheless, for future research, it may be worth investigating the issue of mixtures of decision-making processes in the context of the DDM (cf. Ratcliff, 2006).

There are a number of practical and theoretical implications of our results. First, we showed that deadline-based and cuebased manipulations do not induce a speed-accuracy trade-off in the same way. Because the two manipulations target qualitatively different cognitive processes, the experimenter's choice between the two methods should be carefully considered in studies aimed at experimentally inducing a speedaccuracy trade-off. Second, we provided evidence in favor of the use of accumulation models with collapsing bounds for the study of time pressure effects in perceptual decisionmaking. Collapsing threshold models may provide a more complete representation of how humans respond when faced with particular types of time pressure. Third, we found mixed 
support for the necessity of incorporating variability parameters in the DDM to explain behavior under time pressure: models without non-decision time and starting point variability parameters explained our data better (in contrast to the findings of Rae et al. 2014), but variability in the drift rate was necessary (in line with Voskuilen et al. 2016). These results indicate the need for further research on the set of variability parameters that are necessary to explain the cognitive processes involved in decisions under time pressure. We note that while our results have implications for a range of tasks and evidence accumulation models of perceptual decisionmaking, future investigations should evaluate whether the same computational principles of the SAT described in this study generalize to more complex forms of decision-making (e.g., value-based choices).

Our results regarding decision-making under time pressure are in line with recent literature on the topic. First, we provide empirical evidence supporting Frazier and Yu's (2008) thesis, which predicted that when humans are faced with deadlines, decision-makers require less evidence to commit to a decision as time passes. This is in line with previous work suggesting that decisions for which not enough evidence can be obtained in the allotted time are characterized by collapsing thresholds (Boehm et al. 2016; Evans et al. in press; Hawkins et al. 2015a; Hawkins et al. 2015b; Miletić and van Maanen 2019; Murphy et al. 2016). It is important to note, however, that our modeling analyses did not address the question of whether people followed an optimal or a suboptimal decision policy. This remains a matter of research to be established in the future, though the interested reader is referred to Karşılar et al. (2014) for an earlier comprehensive treatment of the topic.

In the evidence accumulation models we studied here, we accounted for deadline-based time pressure with linearly collapsing thresholds for reasons of simplicity. However, many different functional forms of this kind of urgency signal have been proposed, and the current study did not aim to differentiate between those. In particular, some researchers have argued for an additive (Cisek et al. 2009) or multiplicative temporal signal (Drugowitsch et al. 2012; Frazier and Yu 2008) on the accumulation process (i.e., the drift rate), while others have argued for a concave collapsing threshold (Ratcliff and Frank 2012). However, in terms of response time and accuracy distributions, these proposals are difficult to disentangle, and for that reason we chose the simplest possible functional form with the primary aim of differentiating between conditions without an urgency signal (i.e., a fixed threshold) and conditions with urgency in a model specification that is wellidentified in data (Evans et al. 2019). Although a DDM with linearly collapsing thresholds was able to capture many of the empirical patterns elicited by deadline-based time pressure (yet not perfectly, see, e.g., Fig. 4), it may be that evidence accumulation models with more complex functional forms for the decreasing threshold will further improve the explanation of performance under these conditions. Therefore, further research is required to establish under which conditions more complex functional forms are necessary to explain the behavioral patterns, which might also include other SAT manipulations that do not explicitly use deadlines (e.g., Palestro et al. 2018).

Funding Information Dimitris Katsimpokis was supported by the Onassis Foundation Scholarship Program for Hellenes. Guy Hawkins was supported by an Australian Research Council Discovery Early Career Researcher Award (DE170100177) and Discovery Project (DP180103613).

Open Access This article is licensed under a Creative Commons Attribution 4.0 International License, which permits use, sharing, adaptation, distribution and reproduction in any medium or format, as long as you give appropriate credit to the original author(s) and the source, provide a link to the Creative Commons licence, and indicate if changes were made. The images or other third party material in this article are included in the article's Creative Commons licence, unless indicated otherwise in a credit line to the material. If material is not included in the article's Creative Commons licence and your intended use is not permitted by statutory regulation or exceeds the permitted use, you will need to obtain permission directly from the copyright holder. To view a copy of this licence, visit http://creativecommons.org/licenses/by/4.0/.

\section{References}

Akaike, H. (1974). A new look at the statistical model identification. IEEE Transactions on Automatic Control, 19(6), 716-723. https:// doi.org/10.1109/TAC.1974.1100705.

Ardia, D., Boudt, K., Carl, P., Mullen, K., Peterson, B., Carl, P., Peterson, B. G. (2011). Differential Evolution with DEoptim: An Application to Non-Convex Portfolio Optimization. The R Journal, 3(2), 27-34. https://papers.ssrn.com/sol3/papers.cfm?abstract id=1584905

Ball, K., \& Sekuler, R. (1982). A specific and enduring improvement in visual motion discrimination. Science, 218(4573) Retrieved from http://science.sciencemag.org/content/218/4573/697.

Bhatia, S. (2013). Associations and the accumulation of preference. Psychological Review, 120(3), 522-543. https://doi.org/10.1037/ a0032457.

Boehm, U., Hawkins, G. E., Brown, S., van Rijn, H., \& Wagenmakers, E.-J. (2016). Of monkeys and men: Impatience in perceptual decision-making. Psychonomic Bulletin \& Review, 23(3), 738-749. https://doi.org/10.3758/s13423-015-0958-5.

Boehm, U., Annis, J., Frank, M., Hawkins, G., Heathcote, A., Kellen, D., et al. (2018). Estimating across-trial variability parameters of the diffusion decision model: Expert advice and recommendations. Journal of Mathematical Psychology, 87, 46-75. https://doi.org/ 10.1016/j.jmp.2018.09.004.

Bogacz, R., Brown, E. T., Moehlis, J., Holmes, P., \& Cohen, J. D. (2006). The physics of optimal decision making: A formal analysis of models of performance in two-alternative forced-choice tasks. Psychological Review, 113, 700-765. https://doi.org/10.1037/ 0033-295X.113.4.700.

Bogacz, R., Wagenmakers, E. J., Forstmann, B. U., \& Nieuwenhuis, S. (2010). The neural basis of the speed-accuracy tradeoff. Trends in Neurosciences, 33(1), 10-16. https://doi.org/10.1016/j.tins.2009.09. 002.

Brown, S., Steyvers, M., \& Wagenmakers, E.-J. (2009). Observing evidence accumulation during multi-alternative decisions. Journal of 
Mathematical Psychology, 53(6), 453-462. https://doi.org/10.1016/ J.JMP.2009.09.002.

Busemeyer, J. R., Gluth, S., Rieskamp, J., \& Turner, B. M. (2019). Cognitive and neural bases of multi-attribute, multi-alternative, value-based decisions. Trends in Cognitive Sciences, 23(3), 251263. https://doi.org/10.1016/J.TICS.2018.12.003.

Chandrasekaran, C., \& Hawkins, G. E. (2019). ChaRTr: An R toolbox for modeling choices and response times in decision-making tasks. Journal of Neuroscience Methods, 328, 108432. https://doi.org/10. 1016/J.JNEUMETH.2019.108432.

Cisek, P., Puskas, G. A., \& El-Murr, S. (2009). Decisions in changing conditions: The urgency-gating model. Journal of Neuroscience, 29(37) Retrieved from http://www.jneurosci.org/content/29/37/ 11560.short.

Cox, G. E., \& Shiffrin, R. M. (2017). A dynamic approach to recognition memory. Psychological Review, 124(6), 795-860.

Drugowitsch, J., Moreno-Bote, R., Churchland, A. K., Shadlen, M. N., \& Pouget, A. (2012). The cost of accumulating evidence in perceptual decision making. The Journal of neuroscience : the official journal of the Society for Neuroscience, 32(11), 3612-3628. https://doi.org/ 10.1523/JNEUROSCI.4010-11.2012.

Evans, N. J., \& Hawkins, G. E. (2019). When humans behave like monkeys: Feedback delays and extensive practice increase the efficiency of speeded decisions. Cognition, 184, 11-18. https://doi.org/10. 1016/J.COGNITION.2018.11.014.

Evans, N. J., Hawkins, G. E., Boehm, U., Wagenmakers, E.-J., \& Brown, S. D. (2017). The computations that support simple decision-making: A comparison between the diffusion and urgency-gating models. Scientific Reports, 7(1), 16433. https://doi.org/10.1038/ s41598-017-16694-7.

Evans, N. J., Trueblood, J. S., \& Holmes, W. R. (2019). A parameter recovery assessment of time-variant models of decision-making. Behavior Research Methods, 1-14. https://doi.org/10.3758/ s13428-019-01218-0.

Evans, N. J., Hawkins, G. E., \& Brown, S. D. (in press). The role of passing time in decision-making. Journal of Experimental Psychology: Learning, Memory, and Cognition 1.

Falmagne, J. C. (1968). Note on a simple fixed-point property of binary mixtures. British Journal of Mathematical and Statistical Psychology, 21(1), 131-132. https://doi.org/10.1111/j.2044-8317. 1968.tb00403.x

Forstmann, B. U., Dutilh, G., Brown, S., Neumann, J., von Cramon, D. Y., Ridderinkhof, K. R., \& Wagenmakers, E.-J. (2008). Striatum and pre-SMA facilitate decision-making under time pressure. Proceedings of the National Academy of Sciences of the United States of America, 105(45), 17538-17542. https://doi.org/10.1073/ pnas.0805903105.

Forstmann, B. U., Anwander, A., Schäfer, A., Neumann, J., Brown, S., Wagenmakers, E.-J., Bogacz, R., \& Turner, R. (2010). Corticostriatal connections predict control over speed and accuracy in perceptual decision making. Proceedings of the National Academy of Sciences of the United States of America, 107(36), 15916-15920. https://doi.org/10.1073/pnas.1004932107.

Forstmann, B. U., Ratcliff, R., \& Wagenmakers, E.-J. (2016). Sequential sampling models in cognitive neuroscience: Advantages, applications, and extensions. Annual Review of Psychology, 67.

Frazier, P., \& Yu, A. (2007). Sequential hypothesis testing under stochastic deadlines. In Proceedings of the 20th international conference on neural information processing systems (pp. 465-472) Retrieved from http://dl.acm.org/citation.cfm?id=2981621.

Frazier, P., \& Yu, A. J. (2008). Sequential hypothesis testing under stochastic deadlines. Advances in Neural Information Processing Systems, 20, 465-472.

Gold, J. I., \& Shadlen, M. N. (2007). The neural basis of decision making. Annual Review of Neuroscience, 30(1), 535-574. https://doi.org/10. 1146/annurev.neuro.29.051605.113038.
Hale, D. J. (1969). Speed-error tradeoff in a three-choice serial reaction task. Journal of Experimental Psychology, 81(3), 428-435. https:// doi.org/10.1037/h0027892.

Hawkins, G. E., Brown, S. D., Steyvers, M., \& Wagenmakers, E. (2012). Context effects in multi-alternative decision making: Empirical data and a Bayesian model. Cognitive Science, 36(3), 498-516. https:// doi.org/10.1111/j.1551-6709.2011.01221.x.

Hawkins, G. E., Forstmann, B. U., Wagenmakers, E.-J., Ratcliff, R., \& Brown, S. D. (2015a). Revisiting the evidence for collapsing boundaries and urgency signals in perceptual decision-making. Journal of Neuroscience, 35, 2476-2484.

Hawkins, G. E., Wagenmakers, E.-J., Ratcliff, R., \& Brown, S. (2015b). Discriminating evidence accumulation from urgency signals in speeded decision making. Journal of Neurophysiology, 114(1) Retrieved from http://jn.physiology.org/content/114/1/40.

Heathcote, A., Brown, S. D., \& Wagenmakers, E.-J. (2015). An introduction to good practices in cognitive modeling. In An Introduction to Model-Based Cognitive Neuroscience (pp. 25-48). https://doi.org/ 10.1007/978-1-4939-2236-9 2.

Heitz, R. P. (2014). The speed-accuracy tradeoff: History, physiology, methodology, and behavior. Frontiers in Neuroscience, 8, 150. https://doi.org/10.3389/fnins.2014.00150.

Heitz, R. P., \& Schall, J. D. (2012). Neural mechanisms of speed-accuracy tradeoff. Neuron, 76(3), 616-628. https://doi.org/10.1016/j.neuron. 2012.08.030.

Howell, W. C., \& Kreidler, D. L. (1963). Information processing under contradictory instructional sets. Journal of Experimental Psychology, 65(1), 39-46. https://doi.org/10.1037/h0038982.

Karșilar, H., Simen, P., Papadakis, S., \& Balci, F. (2014). Speed accuracy trade-off under response deadlines. Frontiers in Neuroscience, 8 , 248. https://doi.org/10.3389/fnins.2014.00248.

Malhotra, G., Leslie, D. S., Ludwig, C. J. H., \& Bogacz, R. (2017). Overcoming indecision by changing the decision boundary. Journal of Experimental Psychology: General, 146(6), 776-805. https://doi.org/10.1037/xge0000286.

Miletić, S., \& van Maanen, L. (2019). Caution in decision-making under time pressure is mediated by timing ability. Cognitive Psychology, 110, 16-29. https://doi.org/10.1016/J.COGPSYCH.2019.01.002.

Mulder, M. J., Keuken, M. C., van Maanen, L., Boekel, W., Forstmann, B. U., \& Wagenmakers, E.-J. (2013). The speed and accuracy of perceptual decisions in a random-tone pitch task. Attention, Perception, \& Psychophysics, 75(5), 1048-1058. https://doi.org/ 10.3758/s13414-013-0447-8.

Mulder, M. J., van Maanen, L., \& Forstmann, B. U. (2014). Perceptual decision neurosciences - A model-based review. Neuroscience, 277, 872-884. https://doi.org/10.1016/j.neuroscience.2014.07.031.

Murphy, P. R., Boonstra, E., \& Nieuwenhuis, S. (2016). Global gain modulation generates time-dependent urgency during perceptual choice in humans. Nature Communications, 7(May), 13526. https://doi.org/10.1038/ncomms13526.

Ollman, R. T. (1966). Fast guesses in choice reaction time. Psychonomic Science, 6(4), 155-156. https://doi.org/10.3758/BF03328004.

Palestro, J. J., Weichart, E., Sederberg, P. B., \& Turner, B. M. (2018). Some task demands induce collapsing bounds: Evidence from a behavioral analysis. Psychonomic Bulletin \& Review, 25(4), 12251248. https://doi.org/10.3758/s13423-018-1479-9.

Palminteri, S., Wyart, V., \& Koechlin, E. (2017). The importance of falsification in computational cognitive modeling. Trends in Cognitive Sciences, 21(6), 425-433. https://doi.org/10.1016/j.tics. 2017.03.011.

Peirce, J. W. (2007). PsychoPy-Psychophysics software in python. Journal of Neuroscience Methods, 162(1), 8-13. https://doi.org/10. 1016/j.jneumeth.2006.11.017.

Pike, A. R. (1968). Latency and relative frequency of response in psychophysical discrimination. British Journal of Mathematical and 
Statistical Psychology, 21(2), 161-182. https://doi.org/10.1111/j. 2044-8317.1968.tb00407.x.

Pike, A. R., \& Dalgleish, L. (1982). Latency-probability curves for sequential decision models: A comment on Weatherburn. Psychological Bulletin, 91(2), 384-388. https://doi.org/10.1037/ 0033-2909.91.2.384.

Rae, B., Heathcote, A., Donkin, C., Averell, L., \& Brown, S. (2014). The hare and the tortoise: Emphasizing speed can change the evidence used to make decisions. Journal of Experimental Psychology: Learning, Memory, and Cognition, 40(5), 1226-1243. https://doi. org/10.1037/a0036801.

Ratcliff, R. (2006) Modeling response signal and response time data 2 . Cognitive Psychology 53 (3):195-237

Ratcliff, R. (1978). A theory of memory retrieval. Psychological Review, $85,59-108$

Ratcliff, R., \& Frank, M. J. (2012). Reinforcement-based decision making in corticostriatal circuits: Mutual constraints by neurocomputational and diffusion models. Neural Computation, 24, 1186-1229. https://doi.org/10.1162/NECO_a_00270.

Ratcliff, R., \& McKoon, G. (2008). The diffusion decision model: Theory and data for two-choice decision tasks. Neural Computation, 20(4), 873-922. https://doi.org/10.1162/neco.2008.12-06-420.

Ratcliff, R., \& Rouder, J. N. (1998). Modeling response times for twochoice decisions. Psychological Science, 9(5), 347-356. https://doi. org/10.1111/1467-9280.00067.

Ratcliff, R., \& Rouder, J. N. (2000). A diffusion model account of masking in two-choice letter identification. Journal of Experimental Psychology. Human Perception and Performance, 26(1), 127-140 Retrieved from http://www.ncbi.nlm.nih.gov/ pubmed/10696609.

Ratcliff, R., \& Smith, P. L. (2004). A comparison of sequential sampling models for two-choice reaction time. Psychological Review, 111(2), 333-367.

Ratcliff, R., Smith, P. L., Brown, S. D., \& McKoon, G. (2016). Diffusion decision model: Current issues and history. Trends in Cognitive Sciences, 20(4), 260-281. https://doi.org/10.1016/j.tics.2016.01. 007.

Reppert, T. R., Servant, M., Heitz, R. P., \& Schall, J. D. (2018). Neural mechanisms of speed-accuracy tradeoff of visual search: Saccade vigor, the origin of targeting errors, and comparison of the superior colliculus and frontal eye field. Journal of Neurophysiology, 120(1), 372-384. https://doi.org/10.1152/jn.00887.2017.

Roe, R. M., Busemeyer, J. R., \& Townsend, J. T. (2001). Multialternative decision field theory: A dynamic connectionst model of decision making. Psychological Review, 108(2), 370-392. https://doi.org/ 10.1037/0033-295X.108.2.370.

Scase, M. O., Braddick, O. J., \& Raymond, J. E. (1996). What is noise for the motion system? Vision Research, 36(16), 2579-2586 Retrieved from http://www.ncbi.nlm.nih.gov/pubmed/8917818.

Schouten, J. F., \& Bekker, J. A. M. (1967). Reaction time and accuracy. Acta Psychologica, 27, 143-153. https://doi.org/10.1016/00016918(67)90054-6.

Schwarz, G. (1978). Estimating the dimension of a model. Annals of Statistics, 6, 461-464.

Smith, P. L., \& Vickers, D. (1989). Modeling evidence accumulation with partial loss in expanded judgment. Journal of Experimental
Psychology: Human Perception and Performance, 15(4), 797815. https://doi.org/10.1037/0096-1523.15.4.797.

Thura, D., Beauregard-Racine, J., Fradet, C.-W., \& Cisek, P. (2012). Decision making by urgency gating: Theory and experimental support. Journal of Neurophysiology, 108(11) Retrieved from http://jn. physiology.org/content/108/11/2912.short.

Trueblood, J. S., Brown, S. D., \& Heathcote, A. (2014). The multiattribute linear ballistic accumulator model of context effects in multialternative choice. Psychological Review, 121(2), 179-205. https://doi.org/10.1037/a0036137.

Turner, B. M., Schley, D. R., Muller, C., \& Tsetsos, K. (2018). Competing theories of multialternative, multiattribute preferential choice. Psychological Review, 125(3), 329-362. https://doi.org/10.1037/ rev0000089.

van Maanen, L. (2016). Is there evidence for a mixture of processes in speed-accuracy trade-off behavior? Topics in Cognitive Science, 8(1), 279-290. https://doi.org/10.1111/tops.12182.

Van Maanen, L., Brown, S. D., Eichele, T., Wagenmakers, E.-J., Ho, T. C., Serences, J. T., \& Forstmann, B. U. (2011). Neural correlates of trial-to-trial fluctuations in response caution. Journal of Neuroscience, 31, 17488-17495.

van Maanen, L., de Jong, R., \& van Rijn, H. (2014). How to assess the existence of competing strategies in cognitive tasks: A primer on the fixed-point property. PLoS One, 9(8), e106113. https://doi.org/10. 1371/journal.pone.0106113.

van Maanen, L., Couto, J., \& Lebreton, M. (2016). Three boundary conditions for computing the fixed-point property in binary mixture data. PLoS One, 11(11). https://doi.org/10.1371/journal.pone. 0167377.

Van Zandt, T., Colonius, H., \& Proctor, R. W. (2000). A comparison of two response time models applied to perceptual matching. Psychonomic Bulletin \& Review, 7(2), 208-256. https://doi.org/10. 3758/BF03212980.

Voskuilen, C., Ratcliff, R., \& Smith, P. L. (2016). Comparing fixed and collapsing boundary versions of the diffusion model. Journal of Mathematical Psychology, 73, 59-79. https://doi.org/10.1016/j. jmp.2016.04.008.

Voss, A., Rothermund, K., \& Voss, J. (2004). Interpreting the parameters of the diffusion model: An empirical validation. Memory \& Cognition, 32(7), 1206-1220. https://doi.org/10.3758/BF03196893.

Wagenmakers, E.-J., Ratcliff, R., Gomez, P., \& McKoon, G. (2008). A diffusion model account of criterion shifts in the lexical decision task. Journal of Memory and Language, 58(1), 140-159. https:// doi.org/10.1016/j.jml.2007.04.006.

Wickelgren, W. A. (1977). Speed-accuracy tradeoff and information processing dynamics. Acta Psychologica, 41(1), 67-85. https://doi.org/ 10.1016/0001-6918(77)90012-9.

Winkel, J., Keuken, M. C., van Maanen, L., Wagenmakers, E.-J., \& Forstmann, B. U. (2014). Early evidence affects later decisions: Why evidence accumulation is required to explain response time data. Psychonomic Bulletin \& Review, 21(3), 777-784. https://doi. org/10.3758/s13423-013-0551-8.

Publisher's Note Springer Nature remains neutral with regard to jurisdictional claims in published maps and institutional affiliations. 